\title{
The Information System "Remote Monitoring of Kamchatka and Kuril Islands Volcanic Activity" (VolSatView): Capabilities and Experience
}

\author{
Olga A. Girina ${ }^{1}$, Evgeny A. Loupian ${ }^{2}$, Lubov S. Kramareva ${ }^{3}$, Dmitry V. Melnikov ${ }^{1}$, \\ Alexander G.Manevich ${ }^{1}$, Alexey A. Sorokin ${ }^{4}$, Evgeny I. Gordeev ${ }^{1}$, Ivan A. Uvarov ${ }^{2}$, \\ Alexandr V. Kashnitskii ${ }^{2}$, Mikhail A. Burtsev ${ }^{2}$, Viktor V. Marchenkov ${ }^{2}$, Alexey A. Mazurov ${ }^{2}$, Anna M. \\ Konstantinova ${ }^{2}$, Iraida M. Romanova ${ }^{1}$, Sergey I. Malkovsky ${ }^{4}$, Sergey P. Korolev ${ }^{4}$ \\ ${ }^{1}$ Institute of Volcanology and Seismology FEB RAS, Petropavlovsk-Kamchatsky, Russia \\ girina@kscnet.ru \\ ${ }^{2}$ Space Research Institute RAS, Moscow, Russia \\ evgeny@d902.iki.rssi.ru \\ ${ }^{3}$ Far Eastern Center of "SIC "Planeta", Khabarovsk, Russia \\ ${ }^{4}$ Computing Center FEB RAS, Khabarovsk, Russia
}

\begin{abstract}
The information system "Remote monitoring of Kamchatka and Kuril Islands volcanic activity" (VolSatView IS) was created in 2011. The system provides specialists with access to a variety of information, including long-term archives of remote sensing data needed for remote monitoring of volcanic activity, requiring only a web browser to use the system. Since the launch of the system, the list of available data, as well as tools for their analysis, has been continuously expanding. By now, there is a lot of experience in daily use of the system by the specialists in volcanology. This article describes the current state of the system, including recent developments, such as determination of the height of ash plumes, improvement of the time series analysis tools, implementation of specialized data processing products.
\end{abstract}

Keywords: volcano, Kamchatka, Kuril Islands, monitoring, VolSatView, satellite monitoring, information system, Earth remote sensing

Copyright $₫ 2019$ by the paper's authors. Copying permitted for private and academic purposes 


\title{
ИНФОРМАЦИОННАЯ СИСТЕМА "ДИСТАНЦИОННЫЙ МОНИТОРИНГ АКТИВНОСТИ ВУЛКАНОВ КАМЧАТКИ И КУРИЛ" (ИС VolSatView): ВОЗМОЖНОСТИ И ОПЫТ РАБОТЫ
}

\author{
О.А. Гирина ${ }^{1}$, Е.А. Лупян ${ }^{2}$, Л.С. Крамарева ${ }^{3}$, Д.В. Мельников ${ }^{1}$, А.Г. Маневич ${ }^{1}$, А.А. Сорокин ${ }^{4}$, Е.И. \\ Гордеев ${ }^{1}$, И.А. Уваров ${ }^{2}$, А.В. Кашниикий ${ }^{2}$ М.А. Буриеве ${ }^{2}$, В.В. Марченков ${ }^{2}$, \\ А.А. Мазуров ${ }^{2}$, А.М. Константинова ${ }^{2}$, И.М. Романова ${ }^{1}$, С.И. Мальковский ${ }^{4}$ С.П. Королев ${ }^{4}$ \\ ${ }^{1}$ Институт вулканологии и сейсмологии ДВО РАН, Петропавловск-Камчатский, Россия \\ girina@kscnet.ru \\ ${ }^{2}$ Институт космических исследований РАН, Москва, Россия \\ evgeny@d902.iki.rssi.ru \\ 3 Дальневосточный центр НИЦ "Планета", Хабаровск, Россия \\ ${ }^{4}$ Вычислительный центр ДВО РАН, Хабаровск, Россия
}

В 2011 году была создана информационная система “Дистанционный мониторинг активности вулканов Камчатки и Курил (ИC VolSatView)". Эта система предоставляет специалистам доступ к различной информации, включая долговременные архивы данных Д33, необходимой для решения задач дистанционного мониторинга вулканической активности, при этом требуется лишь наличие web-браузера. C момента запуска системы непрерывно расширялся перечень доступных в ней данных, а также инструментов их анализа. К настоящему времени накоплен опыт ежедневного использования системы специалистами-вулканологами. Настоящая статья рассказывает об актуальном состоянии системы, включая такие новые разработки как определение высоты пепловых шлейфов, развитие инструментов анализа временных рядов данных, создание специализированных продуктов обработки данных.

Ключевые слова: вулкан, Камчатка, Курилы, мониторинг, VolSatView, спутниковый мониторинг, информационная система, дистанционное зондирование Земли

\section{Введение}

На Камчатке и Северных Курилах расположено 36 действующих вулканов [1]. Согласно данным Камчатской группы реагирования на вулканические извержения (KVERT - Kamchatkan Volcanic Eruption Response Team), (http://www.kscnet.ru/ivs/kvert/), на Камчатке ежегодно происходят умеренной силы эксплозивные, эффузивные и экструзивные извержения от 2 до 6 вулканов продолжительностью от нескольких часов до нескольких лет [1], [2]. Эксплозивные извержения вулканов наиболее опасны для населения и авиации, т.к. эруптивные колонны могут подниматься до 35 км н.у.м., пепловые облака и шлейфы перемещаться на тысячи километров, 
пеплопады распространяться на огромные территории и т.д. Наибольшую часть информации о таких событиях можно получить только дистанционными методами.

Спутниковый мониторинг вулканов проводится KVERT с 2002 г. [1]. С созданием в 2011 г. информационной системы (ИС) “Дистанционный мониторинг активности вулканов Камчатки и Курил (VolSatView)” [3] общими усилиями специалистов ИВиС ДВО РАН, ИКИ РАН, ВЦ ДВО РАН и ДВ НИЦ "Планета" вулканологи получили не имеющие аналогов в мире возможности спутниковых наблюдений за активными вулканами. ИC VolSatView, объединяя широкий спектр различных спутниковых данных, предоставляемых ДВ НИЦ Планета и Центром Коллективного Пользования (ЦКП) ИКИ-Мониторинг [4], позволяет непосредственно в web-интерфейсе с помощью созданных специальных инструментов совместно обрабатывать оперативную и ретроспективную (архивы данных более чем за 20 лет) спутниковую информацию (в том числе с 2016 г. данные со спутника Himawari-8, обновляемые каждые 10 минут), сопоставлять еe с видеоинформацией (данные с 10 видеокамер, направленных на вулканы Камчатки), выполнять моделирование распространения пепловых шлейфов, классифицировать различные вулканогенные объекты и т.д.

Основные возможности системы ранее были описаны в работах [3],[5],[6],[7]. Непрерывно расширяются имеющиеся в ней возможности и набор доступных научных данных. Накапливается опыт ежедневного использования системы специалистами-вулканологами. Такой опыт применения и анализа спутниковых и других имеющихся в системе данных при решении реальных задач мониторинга вулканов позволяет постоянно улучшать ее возможности. С момента создания и последнего описания ИС VolSatView в нее были интегрированы новые спутниковые данные, созданы новые информационные продукты, значительно расширены интерактивные инструменты анализа и обработки. Настоящая статья рассказывает об актуальном состоянии системы. Приводится общая схема составных частей ИС VolSatView и их взаимодействия с внешними системами. Рассказано о доступных исследователям данных на текущий момент времени и сделан обзор имеющихся возможностей для их анализа и обработки.

\section{Архитектура построения и основные элементы ИС VolSatView}

ИС VolSatView предоставляет специалистам доступ к большим объемам архивных и оперативных спутниковых и метеорологических данных и результатам их обработки. Получение, обработка и хранение таких данных организовано на базе инфраструктуры различных центров территориально распределенных организаций. В связи с этим, архитектура системы построена таким образом, чтобы обеспечить устойчивую работу с распределенными информационными ресурсами различных центров. Такой подход позволяет пользователям системы через одну точку входа работать с данными, физически находящимися в разных местах. На рисунке показаны основные узлы системы и решаемые каждым узлом задачи, а также потоки обмена данными между ними. Отдельно на схеме изображены взаимодействующие с ИС VolSatView внешние ИС: VOKKIA (Volcanoes of Kurile-Kamchatka Island Arc) [8], KVERT [9] и “Сигнал” [10]. Опишем далее кратко узлы системы и основные их функции.

Центральный информационный узел (ЦИУ), расположенный в ИВиС ДВО РАН (г. Петропавловск-Камчатский) обеспечивает доступ вулканологов к необходимой для мониторинга вулканов информации. В узле хранятся спутниковые данные по территории Камчатки и Северных Курил, поступающие с приемной станции ИВиС ДВО РАН (спутники Terra, Aqua, NPP, JPSS-1), а также в автоматическом режиме передаваемые из других узлов. Этот узел также обеспечивает взаимодействие с ИС VOKKIA и KVERT.

Информационный узел (ИУ) ДЦ НИЦ “Планета” (г. Хабаровск). В этом центре организовано автоматическое получение данных спутника Himawari-8 из Японского агентства аэрокосмических исследований (Japan Aerospace Exploration Agency - JAXA), а также прием данных спутников NOAA, Terra, Aqua, NPP, Метеор-M, Канопус, Ресурс и других.

ИУ ВЦ ДВО РАН (г. Хабаровск) обеспечивает ведение архивов данных, поступающих в систему из ДЦ НИЦ “Планета". Также на базе его ресурсов организовано взаимодействие с 
автоматизированной ИС (АИС) “Сигнал” для обеспечения моделирования траекторий движения пепловых облаков и получения данных видеонаблюдения за вулканами Камчатки.

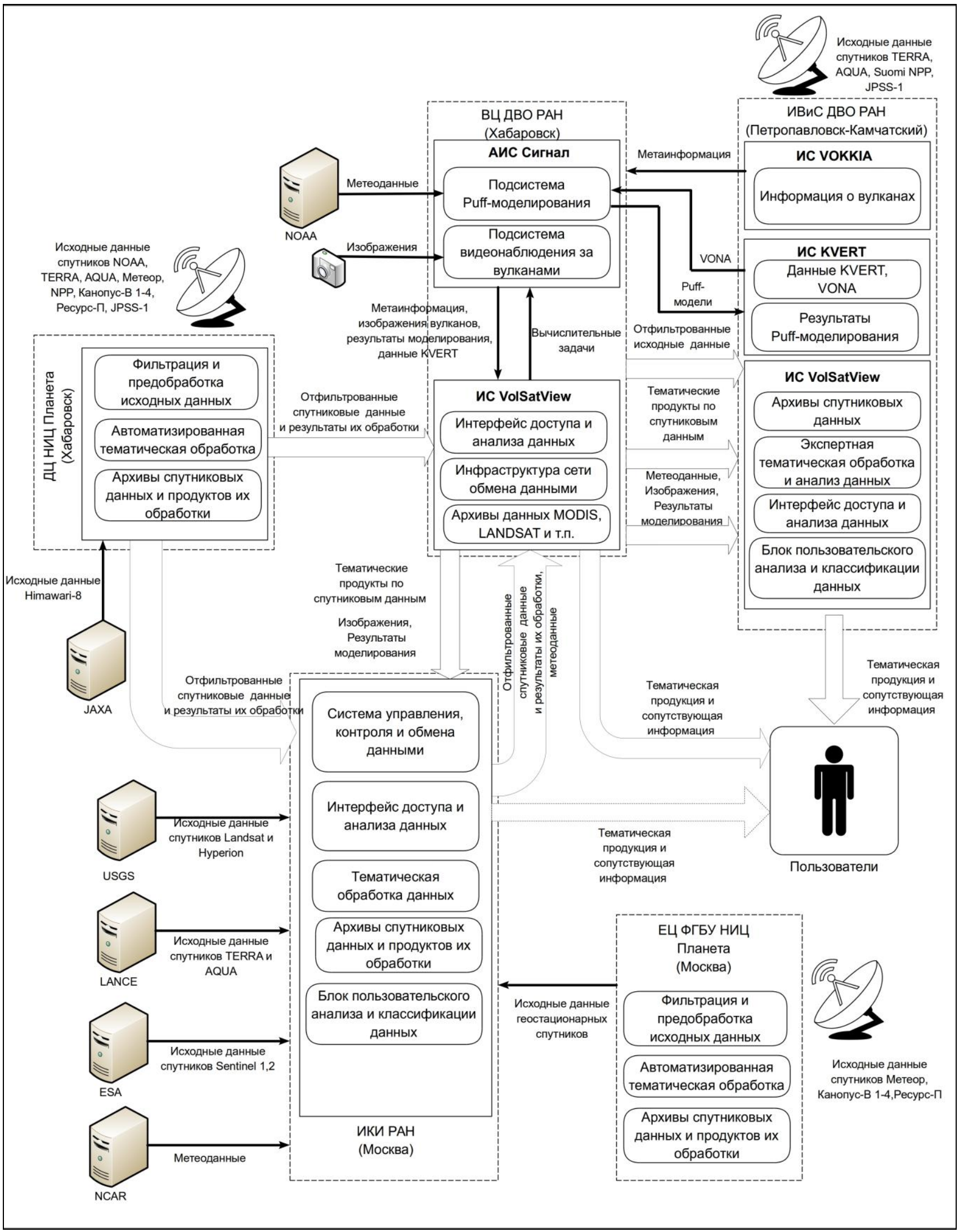

Рисунок. Архитектура построения ИС VolSatView 
ИУ ИКИ РАН (г. Москва) обеспечивает оперативный доступ к данным, предоставляемым информационными сервисами Центра коллективного пользования “ИКИ-Мониторинг” [4]. В этом узле организовано оперативное получение из различных зарубежных и отечественных центров спутниковых данных Landsat, Sentinel 1, Sentinel 2, Метеор-M, Канопус-B, Hyperion, Terra, Aqua, NOAA, NPP и других. Часть этой информации в автоматическом режиме поступает в другие центры. В узле также организовано получение и обработка метеорологической информации.

ИУ ЕЦ ФГБУ НИЦ “Планета” (г. Москва) с помощью возможностей объединенной системы работы со спутниковыми данными центров НИЦ “Планета" предоставляет в ИС VolSatView информацию со спутников Метеор, Канопус-В, Ресурс-П.

\section{Доступные в ИС VolSatView спутниковые данные}

Как уже было сказано, для решения задач мониторинга вулканов в ИС VolSatView организован online доступ к данным большого количества различных установленных на спутниках приборов. Основные типы получаемой в системе информации и задачи, для решения которых она используется, представлены в таблице. Для каждого из указанных приборов пользователям системы предоставляется достаточно большой набор тематических информационных продуктов, полученных в результате обработки исходных инструментальных данных. Такой доступ организован на основе разработанных ранее в ИКИ РАН технологий [11]. Полный список доступных тематических продуктов на конец 2018 года приводился нами в [5]. Особо отметим, что все указанные данные доступны пользователям системы в режиме online, без предварительного их заказа и необходимости скачивания.

\section{Основные возможности анализа данных, реализованные в ИС VolSatView}

Анализ термальных аномалий в районах вулканов возможен на основе данных приборов AVHRR, MODIS, VIIRS как в автоматическом (с использованием алгоритмов MOD14 и MODVOLC), так и интерактивном режиме. При этом производится оценка температуры отдельных точек и фона, а также расчет нормализованного теплового индекса. Оперативное пополнение данных со спутника Himawari-8 обеспечивает возможность анализа временных рядов наблюдения температуры поверхности земли.

Для выделения пепловых шлейфов в системе используется разность радиояркостных температур в каналах 11 и 12 мкм. Задача может быть решена в ручном или полуавтоматическом режиме. В первом случае пользователь определяет шлейф визуально и вводит контур. Во втором случае проводится необучаемая классификация изображения с последующим экспертным контролем результата.

Оценка высоты пепловых облаков и шлейфов выполняется двумя способами. Первый основан на сопоставлении радиояркостной температуры, полученной по спутниковым данным, с вертикальным профилем метеорологических данных. Второй способ совместно использует данные геостационарных и низкоорбитальных спутников, обладающих разными углами наблюдения, позволяющие определить высоту пеплового облака на основе параллакса.

Для анализа динамических процессов в системе предусмотрено построение цветовых синтезов на основе разновременных изображений. Другой подход к исследованию динамики анализ временных рядов данных в виде графиков, включая расчет производных величин, таких как индексы, скользящие средние, фоновая составляющая.

Анализ гиперспектральных данных, в частности, полученных прибором Hyperion, обеспечивает подробное описание характеристик наблюдаемых объектов. Это позволяет, например, изучать состояние пепловых шлейфов, структуры лавовых и пирокластических потоков и вулканогенных пород.

Интеграция системы VolSatView с АИС “Сигнал” обеспечивает совместный анализ результатов моделирования распространения пепловых шлейфов и спутниковых данных. Это 
позволяет исследователю сформировать задание на проведение моделирования конкретного эксплозивного события, передать его в АИС “Сигнал”, а затем визуализировать его результаты с помощью картографического интерфейса VolSatView.

Таблица. Основные виды спутниковой информации в ИC VolSatView

\begin{tabular}{|c|c|c|c|c|}
\hline Диапазон & Разрешение & Прибор (спутник) & $\begin{array}{l}\text { Частота } \\
\text { наблюдения }\end{array}$ & $\begin{array}{l}\text { Основные решаемые } \\
\text { задачи }\end{array}$ \\
\hline \multirow{5}{*}{$\begin{array}{l}\text { Оптика } \\
0,4-0,9 \text { мкм }\end{array}$} & Более 2 км & AHI (Himawari 8 ) & 10 мин & \multirow{5}{*}{$\begin{array}{l}\text { Детектирование } \\
\text { пепловых шлейфов и } \\
\text { оценка их параметров. } \\
\text { Картирование и } \\
\text { определение } \\
\text { характеристик } \\
\text { отложений. }\end{array}$} \\
\hline & 1 км & $\begin{array}{l}\text { MODIS (Terra/Aqua) } \\
\text { AVHRR (NOAA) } \\
\text { VIIRS (Suomi NPP, JPSS1) } \\
\text { MCУ MP (Метеор M) }\end{array}$ & $\begin{array}{l}\text { От } 15 \text { до } 25 \\
\text { в сутки* }\end{array}$ & \\
\hline & $100-300 \mathrm{M}$ & $\begin{array}{l}\text { Terra/Aqua (MODIS) } \\
\text { VIIRS (Suomi NPP, JPSS1) }\end{array}$ & $\begin{array}{l}\text { От } 5 \text { до } 8 \text { в } \\
\text { сутки* }\end{array}$ & \\
\hline & $10-60$ м & $\begin{array}{l}\text { TM, ETM+, OLI (Landsat), } \\
\text { MSI (Sentinel 2A/B) } \\
\text { MCС (серия спутников } \\
\text { "Канопус В"), } \\
\text { КМСС (Метеор М) }\end{array}$ & $\begin{array}{l}\text { От одного } \\
\text { раза в двое } \\
\text { суток до } \\
\text { раза в } \\
\text { сутки* }\end{array}$ & \\
\hline & лучше 10 м & $\begin{array}{l}\text { ПСС (серия спутников } \\
\text { "Канопус В"), } \\
\text { Геотон-П (Ресурс П) } \\
\text { ORBVIEW-3 }\end{array}$ & $\begin{array}{l}\text { Несколько } \\
\text { раз в год по } \\
\text { отдельным } \\
\text { участкам }\end{array}$ & \\
\hline \multirow{4}{*}{$\begin{array}{l}\text { Ближний } \\
\text { ИК } \\
1,4-3,9 \text { мкм }\end{array}$} & Более 2 км & AHI (Himawari 8 ) & 10 мин & \multirow{4}{*}{$\begin{array}{l}\text { Выделение аномалий и } \\
\text { оценка характеристик } \\
\text { температуры. } \\
\text { Анализ характеристик } \\
\text { термальных аномалий. } \\
\text { Анализ характеристик } \\
\text { пепловых облаков и } \\
\text { шлейфов }\end{array}$} \\
\hline & 1 км & $\begin{array}{l}\text { MODIS (Terra, Aqua) } \\
\text { VIIRS (Suomi NPP, JPSS1) } \\
\text { AVHRR (NOAA) } \\
\text { MCУ MP (Метеор M) }\end{array}$ & $\begin{array}{l}\text { От } 15 \text { до } 25 \\
\text { в сутки* }\end{array}$ & \\
\hline & $200-800 \mathrm{M}$ & $\begin{array}{l}\text { VIIRS (Suomi NPP, JPSS1) } \\
\text { МС-ИК-СРМ (Канопус В ИК) }\end{array}$ & $\begin{array}{l}\text { От } 5 \text { до } 8 \text { в } \\
\text { сутки* }\end{array}$ & \\
\hline & $10-30 \mathrm{M}$ & $\begin{array}{l}\text { TIRS (Landsat), } \\
\text { MSI (Sentinel } 2 \text { A/B) }\end{array}$ & $\begin{array}{l}\text { От одного } \\
\text { раза в пять } \\
\text { суток до } \\
\text { раза в трое } \\
\text { суток* }\end{array}$ & \\
\hline \multirow{3}{*}{$\begin{array}{l}\text { ИК } \\
11-12 \text { мкм }\end{array}$} & Более 2 км & AHI (Himawari 8 ) & 10 мин & \multirow{3}{*}{$\begin{array}{l}\text { Выделение аномалий и } \\
\text { оценка характеристик } \\
\text { температуры. } \\
\text { Детектирование } \\
\text { пепловых шлейфов и } \\
\text { оценка их параметров. } \\
\text { Анализ характеристик } \\
\text { термальных аномалий. } \\
\text { Анализ характеристик } \\
\text { пепловых облаков и } \\
\text { шлейфов }\end{array}$} \\
\hline & 1 км & $\begin{array}{l}\text { Terra/Aqua (MODIS) } \\
\text { VIIRS (Suomi NPP, JPSS1) } \\
\text { AVHRR (NOAA), } \\
\text { MCУ MP (Метеор M) }\end{array}$ & $\begin{array}{l}\text { От } 15 \text { до } 25 \\
\text { в сутки* }\end{array}$ & \\
\hline & $\begin{array}{l}200 \mathrm{M} \\
60 \mathrm{M}\end{array}$ & $\begin{array}{l}\text { МСУ-ИК-СМР } \\
\text { TIRS (Landsat), }\end{array}$ & $\begin{array}{l}\text { Раз в 2-3 дня } \\
\text { Раз в } 14 \\
\text { дней }\end{array}$ & \\
\hline $\begin{array}{l}\text { Радиолока- } \\
\text { торы }\end{array}$ & $20 \times 40 \mathrm{M}$ & C_SAR (Sentinel 1A/B) & $\begin{array}{l}1 \text { раз в } 5 \\
\text { дней }\end{array}$ & $\begin{array}{l}\text { Анализ вулканических } \\
\text { отложений. }\end{array}$ \\
\hline $\begin{array}{l}\text { Гиперспектр } \\
\text { ометры }\end{array}$ & $30 \mathrm{M}$ & Hiperion (EO-1) & $\begin{array}{l}\text { Несколько } \\
\text { раз в год }\end{array}$ & $\begin{array}{l}\text { Анализ вулканических } \\
\text { отложений. } \\
\text { Анализ пепловых облаков } \\
\text { и шлейфов }\end{array}$ \\
\hline
\end{tabular}

Примечание. * цифры приведены для районов от Южных Курил до северной части Камчатки 


\section{Заключение}

На сегодняшний день информационная система VolSatView является уникальным инструментом, который позволяет решать задачи разного уровня - от оперативного мониторинга активности вулканов до фундаментальных проблем вулканологии. За последние годы с помощью ИС VolSatView был решен целый ряд различных задач, например, освещенных в работах [5],[12],[13],[14]. Другой пример: в результате ежедневного мониторинга вулканов Камчатки с помощью ИC VolSatView ученые KVERT выпустили в 2017 г. 275 сообщений VONA (Volcano Observatory Notice for Aviation) об опасных эксплозивных извержениях шести вулканов (Шивелуч, Ключевской, Безымянный, Карымский, Жупановский, Камбальный). Возможности ИC VolSatView по мониторингу вулканической активности и изучению вулканов непрерывно будут развиваться и в дальнейшем.

При создании ИC VolSatView использовались ресурсы Центра коллективного пользования “ИКИ-Мониторинг”, работа которого осуществляется при поддержке ФАНО (тема «Мониторинг», госрегистрация №01.20.0.2.00164).

Работа выполнена при поддержке Российского научного фонда (проект № 16-17-00042).

\section{References}

[1] Girina O.A., Melnikov D.V., Manevich A.G. Satellite monitoring of Kamchatkan and Northern Kuriles volcanoes, Sovremennye Problemy Distantsionnogo Zondirovaniya Zemli iz Kosmosa, 2017, 14(6), pp. 194-209. DOI: $10.21046 / 2070-7401-2017-14-6-194-209$

[2] Gordeev E.I., Girina O.A. Volcanoes and their hazard to aviation, Herald of the Russian Academy of Sciences, 2014, 84(1), pp. 1-8. DOI: 10.1134/S1019331614010079.

[3] Gordeev E.I., Girina O.A., Loupian E.A., Sorokin A.A., Kramareva L.S., Efremov V.Yu., Kashnitskii A.V., Uvarov I.A., Burtsev M.A., Romanova I.M., Melnikov D.V., Manevich A.G., Korolev S.P., Verkhoturov A.L. The VolSatView information system for Monitoring the Volcanic Activity in Kamchatka and on the Kuril Islands, Journal of Volcanology and Seismology, 2016, 10(6), pp. 382-394. DOI: 10.1134/S074204631606004X

[4] Loupian E.A., Proshin A.A., Burtsev M.A., Balashov I.V., Bartalev S.A., Efremov V.Yu., Kashnitskiy A.V., Mazurov A.A., Matveev A.M., Sudneva O.A., Sychugov I.G., Tolpin V.A., Uvarov I.A. IKI center for collective use of satellite data archiving, processing and analysis systems aimed at solving the problems of environmental study and monitoring, Sovremennye Problemy Distantsionnogo Zondirovaniya Zemli iz Kosmosa, 2015, 12(5), pp. 263-284 (In Russian).

[5] Girina O.A., Loupian E.A., Sorokin A.A., Melnikov D.V., Romanova I.M., Kashnitskii A.V., Uvarov I.A., Malkovsky S.I., Korolev S.P., Manevich A.G., Kramareva L.S. Comprehensive monitoring of explosive volcanic eruptions of Kamchatka /Ed. O.A. Girina, Petropavlovsk-Kamchatsky: IVS FEB RAS, 192 p. (In Russian)

[6] Gordeev E.I., Girina O.A., Loupian E.A., Sorokin A.A., Melnikov D.V., Manevich A.G., Romanova I.M., Kramareva L.S., Efremov V.Yu., Kobets D.A., Kashnitskii A.V., Korolev S.P., Bourtsev M.A., Samoilenko S.B. Comprehensive analysis of Kamchatkan explosive volcanic eruptions data in VolSatView Information System, Volcanism and associated processes, Proc. Regional Scientific Conf., 29-30 March 2016, PetropavlovskKamchatsky: IVS FEB RAS, 2016, pp. 53-64 (In Russian)

[7] Gordeev E.I., Girina O.A., Loupian E.A., Sorokin A.A., Melnikov D.V., Manevich A.G., Romanova I.M., Kramareva L.S., Efremov V.Yu., Kobets D.A., Kashnitskii A.V., Verkhoturov A.L., Bourtsev M.A., Information system VolSatView for a comprehensive analysis of the Kamchatka volcanic explosive eruptions data, Vestnik Dalnevostochnogo Otdeleniya Rossiiskoi Akademii Nauk, 2016, 5, pp. 397-410 (In Russian)

[8] Romanova I.M., Girina O.A., Maksimov A.P., Melekestsev I.V. Creation of complex information web system Volcanoes of the Kurile-Kamchatka Island Arc (VOKKIA), Informatika i sistemy upravleniya, 2012, 33(3), pp. 179-187 (In Russian) 
[9] Romanova I.M., Girina O.A. Information Technologies for Data Analysis of the Kamchatka and Kuriles Volcanoes // Bulletin KRAUNZ. Earth Sciences, 2018, 39(3), pp. 42-53. DOI: 10.31431/1816-5524-2018-3-39$42-53$

[10] Korolev S.P., Sorokin A.A., Verkhoturov A.L., Konovalov A.V., Shestakov N.V. Automated information system for instrument-data processing of the regional seismic observation network of FEB RAS, Seismic Instruments, 2015, 51(3), pp. 209-218 (In Russian)

[11] Proshin A.A., Loupian E.A., Balashov I.V., Kashnitskii A.V., Bourtsev M.A. Unified satellite data archive management platform for remote monitoring systems development, Sovremennye Problemy Distantsionnogo Zondirovaniya Zemli iz Kosmosa, 2016, 13(3), pp. 9-27. DOI: 10.21046/2070-7401-2016-13-3-9-27

[12] Girina O.A., Kramareva L.S., Loupian E.A., Melnikov D.V., Manevich A.G., Sorokin A.A., Uvarov I.A., Kashnitskii A.V., Burtsev M.A., Marchenkov V.V., Bril’ A.A., Mazurov A.A., Romanova I.M., Malkovskii S.I. The use of Himawari satellite data for monitoring Kamchatka volcanoes, Sovremennye Problemy Distantsionnogo Zondirovaniya Zemli iz Kosmosa, 2017, 14(7), pp. 65-76. DOI: 10.21046/2070-7401-2017-14-765-76

[13] Girina O.A., Loupian E.A., Melnikov D.V., Manevich A.G., Kashnitskii A.V., Bril' A.A., Sorokin A.A. Eruptions of Kamchatka Northern volcanic group on 14-18 June, 2017, Sovremennye Problemy Distantsionnogo Zondirovaniya Zemli iz Kosmosa, 2017, 14(3), pp. 317-323. DOI: 10.21046/2070-7401-2017-14-3-317-323

[14] Girina O.A., Loupian E.A., Melnikov D.V., Manevich A.G., Sorokin A.A., Kramareva L.S., Uvarov I.A., Kashnitskii A.V. Bezymianny volcano eruption on December 20, 2017, Sovremennye Problemy Distantsionnogo Zondirovaniya Zemli iz Kosmosa, 2018, 15(3), pp. 88-99. DOI: 10.21046/2070-7401-2018-15-3-88-99 